PUPT 1480, IASSNS 94/51

hep-th/9408XXXX

August 1994

\title{
Geometric Entropy, Wave Functionals, and Fermions
}

\author{
FINN LARSEN * \\ Department of Physics \\ Joseph Henry Laboratories \\ Princeton University \\ Princeton, N.J. 08544
}

\author{
FRANK WILCZEK ${ }^{\dagger}$ \\ School of Natural Sciences \\ Institute for Advanced Study \\ Olden Lane \\ Princeton, N.J. 08540
}

\footnotetext{
$\star$ Research supported in part by Danish National Science Foundation Fellowship. larsen@puhep1.princeton.edu

$\dagger$ Research supported in part by DOE grant DE-FG02-90ER40542. wilczek@iassns.bitnet
} 


\begin{abstract}
We develop techniques for calculating the ground state wave functional and the geometric entropy for some simple field theories. Special attention is devoted to fermions, which present special technical difficulties in this regard. Explicit calculations are carried through for free mass bosons and fermions in two dimensions, using an adaptation of Unruh's technique to treat black hole radiance.
\end{abstract}




\section{Introduction}

Recently there has been great interest in the concept of geometric entropy [1-9]. Almost all of the explicit work has been done for free scalar bosons, although one can adapt conformal field theory arguments to cover some additional special cases. Among several possible generalizations, perhaps the most interesting is to fermions. Fermions play a role in several of the speculations regarding geometric entropy, especially those involving its analogues in superstring theory. The ground state wave functional and the path integral, fundamental objects from any perspective and certainly central to any discussion of geometric entropy, have quite a different character for fermions than for bosons. Whereas for bosons the states of the quantum field theory can be labelled by classical field configurations, since the field operators on a complete hypersurface constitute a complete set of commuting observables, the corresponding structure for fermions is less transparent.

Let us recall the basic construction used to define geometric entropy. One divides space into two regions, which we shall call inside and outside. We consider a quantum field theory defined in all of space, and possessing a complete set of local observables $\xi(x)$. We divide these into two disjoint subsets according to whether their argument $x$ lies on the inside or on the outside. Any state may be labelled by its amplitude as a function of the the eigenvalues $\xi_{\text {in }}, \xi_{\text {out }}$ of the $\xi(x)$ with $x$ lying on the inside or the outside respectively. Now consider a definite pure state described by the wave functional $\Psi\left[\xi_{\text {in }}, \xi_{\text {out }}\right]$. In the body of this paper, we shall be concerned with the ground state exclusively, and when we speak of geometric entropy without further specification we shall have this case in mind. Then the density matrix appropriate to an observer who only has access to the outside region is obtained by tracing over the variables localized in the in-region:

$$
\rho\left(\xi_{\text {out }}^{1}, \xi_{\text {out }}^{2}\right)=\sum_{\xi_{\text {in }}} \bar{\Psi}\left[\xi_{\text {in }}, \xi_{\text {out }}^{1}\right] \Psi\left[\xi_{\text {in }}, \xi_{\text {out }}^{2}\right]
$$

This density matrix in general no longer describes a pure state, and one defines 
the geometric entropy relative to the given state and the given division of space as

$$
S_{\text {geom }}=-\operatorname{Tr} \rho \ln \rho
$$

This $S$ is a measure of the strength of the correlations between the inside region and the outside region, information concerning which is lost in the process of tracing over the inside region. It has several qualitative properties that are rather different from those of thermodynamic entropy; notably it is not extensive, and its value is invariant under interchange of "inside" with "outside". In field theories with sufficiently singular ultraviolet behavior (that is, in essentially all ordinary non-topological - field theories) the geometric entropy is dominated by short-range correlations. In the ultraviolet limit all theories look scale invariant, and furthermore the important correlations arise from field fluctuations in the direction of the normal at the boundary. As a matter of technical convenience, rather than conceptual necessity we shall, below, often use free massless fields in $1+1$ dimensions as our working material. Due to the considerations just mentioned, this specialization is less severe than might appear at first sight. (In this regard, the interested reader might like to refer to Equation 36 of [4] as an illustration of the special significance of the $1+1$ dimensional massless theories. Such theories, of course, also occupy center stage in superstring theory.)

Although we will not pursue it much further in this paper, let us briefly note that one can also define the geometric entropy for mixed states, e.g. thermal states. In that context several of the points made above require modification. In particular, there is no longer symmetry between inside and outside; futhermore, the difference between the thermal geometric entropies at different temperatures is not expected to be dominated by ultraviolet behavior, and it should be approximately extensive for large volumes - the bulk geometric entropy density coincides with the bulk thermodynamic entropy.

We have considered the geometric entropy in general conformal field theories previously, and obtained quantitative results which coincide with the ones presented here [8]. The goal of the present work is, in a sense, to do it again the hard 
way - working with field variables (especially, fermion field variables ) directly, without invoking conformal symmetry. Our goal is, by being very pedestrian and explicit, to sharpen our understanding of the technical issues involved, particularly in defining the wave functionals and the path integrals.

As an example of the sort of subtlety that arises, consider the following. In their standard formulation, relativistic fermions obey a linear equation, arising from a quadratic action. One is accustomed to evaluating quadratic path integrals by substituting the solutions of the classical equations of motion (with the appropriate boundary conditions) back into the action. But here the action will simply vanish, and by proceeding naively one obtains a trivial - wrong - answer.

Below, by exercising more care, we shall be able to obtain meaningful, useful expressions in this and similar contexts. Having these in hand, we will proceed to evaluate the density matrix and geometric entropy for free massless bosons and fermions in $1+1$ dimensions. When the dust settles, we are left with a very convenient formalism that treats bosons and fermions on an equal footing. Here, as in most other work on geometric entropy, the insight that allows us to pursue matters to the very end is an inspired choice of variables, first used by Unruh [10], who used it in a different but related context. But there are some small surprises in the details: our generalization of the Unruh ansatz contains a funny coefficient, signs must be treated very carefully, and boundary conditions are nontrivial.

\section{The Wave Functional}

To discuss geometric entropy we need to describe our reference state in terms of local variables. In ordinary quantum mechanics we can project on eigenstates $|q\rangle$ of the position operator $\hat{q}$, thus defining $\Psi(q, t)=\langle q \mid \Psi(t)\rangle$. For field theory, we want to choose a complete set of local variables generalizing $\hat{q}$. The field operators $\hat{\phi}(x)$ form a natural choice, but it is by no means unique. We might, for example, choose instead to diagonalize the canonical momenta $\hat{\pi}_{\phi}(x)$. It is by no means 
obvious at first sight what constitutes the "natural" choice is for fermions; we shall see it is in a sense a linear combination of the two just mentioned.

\subsection{Schrödinger Equation And Path Integral}

Although the definition of geometric entropy does not refer explicitly to dynamics, we will find it useful to consider solutions to the time-dependent Schrödinger equation

$$
i \frac{d}{d t}|\Psi\rangle=\hat{H}|\Psi\rangle
$$

where $\hat{H}$ is the Hamiltonian operator. The formal solution is

$$
\left|\Psi\left(t_{f}\right)\right\rangle=T e^{-i \hat{H}\left(t_{f}-t_{i}\right)}\left|\Psi\left(t_{i}\right)\right\rangle
$$

where $\mathrm{T}$ denotes time-ordering. Time-ordering is an essential requirement, because the Hamiltonian is an integral over objects that do not mutually commute.

The formal expression (2.2) for the wave function is difficult to evaluate explicitly for specific field theories, and the time-ordering obscures the Lorentz invariance of the theory. Indeed, the Hamiltonian as well as the time-ordering procedure depend explicitly on the choice of reference frame. Fortunately, one has the identity

$$
\Psi\left[\phi_{f}\right]=\left\langle\phi_{f}\left|T e^{-i \hat{H}\left(t_{f}-t_{i}\right)}\right| \phi_{i}\right\rangle=\int \mathcal{D} \phi e^{i A(\phi)}
$$

In this path integral, one integrates over all field configurations for which the field is a prescribed value $\phi_{i}$ at some early time $t_{i}$ and equal to $\phi_{f}$ at the final time $t_{f}$. The field configuration at the early time selects a specific state. The measure will be constructed below. If the action $A(\phi)$ and the measure $\mathcal{D} \phi$ are Lorentz invariant, the transformation law for the wave functional is simply given by appropriate change in boundary conditions. 


\subsection{Symmetry And the Geometric Derivation}

We recall a recent simple derivation [11] of (2.3), that is very instructive for our purposes. The Hamiltonian expression satisfies a simple first order differential equation. Since both expressions reduce to 1 for $t_{f}=t_{i}$, to prove their identity it suffices to show that the Lagrangian expression satisfies the same differential equation. Thus we must evaluate

$$
\delta \int \mathcal{D} \phi e^{i \int_{t_{i}}^{t_{f}} \mathcal{L}(\phi) d^{d} x}
$$

where the variation is a change in $t_{f}$ to $t_{f}+\delta t_{f}$.

The definition of the energy momentum tensor $T_{\mu \nu}$ is

$$
d A=-\frac{1}{4 \pi} \int T_{\mu \nu} d g^{\mu \nu} d^{d} x
$$

under a change $d g^{\mu \nu}$ in metric. The variation $\delta$ may be implemented by choosing a new $g_{00}=\left(1+2 \frac{\delta t_{f}}{t_{f}-t_{i}}+\right.$ const). Following [11] (using, in essence, a special case of the Schwinger action principle), we require the expression to be invariant under changes in coordinates, thus deriving

$$
\delta \int \mathcal{D} \phi e^{i A} \simeq \frac{i}{4 \pi} \int \mathcal{D} \phi e^{i A} \int T_{\mu \nu} d g^{\mu \nu} d^{d} x
$$

for true changes in geometry. Notice the sign of this expression. Next, we can use conservation of (integrated!) energy-momentum to put this in the form

$$
\frac{\delta \int \mathcal{D} \phi e^{i A}}{\delta t_{f}}=-\frac{i}{2 \pi} \frac{1}{t_{f}-t_{i}} \int \mathcal{D} \phi\left(\int T_{00} d^{d} x\right) e^{i A}=-i \hat{H} \Psi[\phi],
$$

which establishes (2.3). The action of Hamiltonian operator $\hat{H}$ on the wave functional is defined by the last equation, i.e. it is found by evaluating the operation occurring in (2.6) on the final slice of the functional integral. 
For the preceding derivation, the crucial property of the path integral measure employed is invariance under time dilation. We will also require invariance under translation with a function. A measure satisfying these properties will be constructed in the next sections, and will involve some surprising subtleties.

To motivate the detailed investigation, let us elaborate further upon an difficulty with with the naïve application of the path integral for fermions, that we already touched on briefly. For simplicity, we consider a Weyl fermion in two dimensions. On shell this can be represented as a function of (say) $x+t$, or in complex variables as a holomorphic function of $z$. The action is $A=\frac{1}{2 \pi} \int \psi \bar{\partial} \psi$. Now it appears that, at the saddle point, this action vanishes, since the equation of motion is simply $\bar{\partial} \psi=0$. One the other hand, consider the Hamiltonian expression for the wave functional: the Hamiltonian $\hat{H}=\frac{i}{4 \pi} \int \psi \partial_{\sigma} \psi$ certainly does not vanish for holomorphic $\psi$ s. How, then, can the two expressions (2.3) for the wave functional possibly agree?

To address this question recall that in the derivation of the path integral formula for the wave function we exploited the feature that the measure is invariant under a rescaling of the time variable. But under such a rescaling, $z$ mixes with $\bar{z}$. It is therefore important that, as intermediate configurations in the path integral, we use a set of states that is closed under time translations. That means that even for chiral particles such as Weyl fermions, one cannot impose the equation of motion on intermediate states. To get a simple path integral we must allow both right and left movers in intermediate states, even for a Weyl particle. Once we do that, it becomes less mysterious why the action does not necessarily vanish. Note that in this reasoning we tacitly assumed that a stationary phase method is applicable and exact for the quadratic action, or in other words that by imposing the equations of motions found by varying the action with respect to the field, we find a field $\psi_{\mathrm{cl}}$ that saturates the functional integral. In the next sections we will construct the path integral that has this desirable feature explicitly, and verify that indeed such a classical field exists even for chiral fermions - but the classical field will include both left and right movers. 


\subsection{The Holomorphic Path Integral}

A popular modern way to construct path integrals uses the holomorphic representation, also called the Bargmann-Fock representation [12-13]. In this representation we work in bases that diagonalize the positive and negative frequency parts

of the field, rather than the Hermitean operators $\hat{\phi}$ and $\hat{\pi} \propto \partial_{t} \hat{\phi}$ which are perhaps more natural from an intuitive standpoint. In the holomorphic representation we diagonalize the $\hat{\phi}(x)+i \hat{\pi}(x)$ and thus also their Fourier transforms, denoted $\hat{\phi}_{k}$. It is in terms of the these latter operators that the free Hamiltonian can be expressed as a sum of fundamental harmonic oscillators, i.e. $\hat{H}=\int \frac{d k}{2 \pi} \omega_{k} \hat{\phi}_{k}^{\dagger} \hat{\phi}_{k}$. In principle all we need for the calculation of the geometric entropy (or anything else) is the ability to evaluate a complete set of commuting local variables, so either choice will serve.

For a boson field $\phi$ we use the coherent ket states

$$
|\phi\rangle=\prod_{k} e^{-\frac{1}{2} \bar{\phi}_{k} \phi_{k}} e^{\hat{\phi}_{k}^{\dagger} \phi_{k}}|0\rangle .
$$

This state is an eigenfunction of the positive frequency part of the field operator, i.e. $\hat{\phi}_{k}|\phi\rangle=\phi_{k}\left|\phi_{k}\right\rangle$. The index $k$ labels the degrees of freedom which, for a free scalar field, can be identified with the momentum. For massless fields in two dimensions $k$ may be restricted to take either only positive or only negative values, if one of these restrictions is imposed the field is chiral. The bras are obtained by complex conjugation of the kets. They diagonalize the negative frequency part of the field to the acting to the left: $\langle\phi| \hat{\phi}_{k}^{\dagger}=\langle\phi| \bar{\phi}_{k}$. There is a completeness relation

$$
\int \prod_{k} d \bar{\phi}_{k} d \phi_{k}|\phi\rangle\langle\phi|=1
$$

which implicitly normalizes the measure. 
Now we can evaluate the evolution operator, as follows:

$$
\begin{aligned}
U\left(\bar{\phi}_{f} \mid \phi_{i}\right) & \equiv\left\langle\bar{\phi}_{f}\left|T e^{-i \hat{H} t}\right| \phi_{i}\right\rangle \\
& =\int \prod_{k}\left(\prod_{j=1}^{n-1} d \bar{\phi}_{j} d \phi_{j}\right) \prod_{j=0}^{n-1}\left\langle\bar{\phi}_{j+1}\left|e^{-i \omega_{k} \hat{\phi}_{k}^{\dagger} \hat{\phi}_{k} \Delta t_{j}}\right| \phi_{j}\right\rangle \\
& =\int \prod_{k}\left(\prod_{j=1}^{n-1} d \bar{\phi}_{j} d \phi_{j}\right) \prod_{j=0}^{n-1}\left\langle\bar{\phi}_{j+1} \mid e^{-i \omega_{k} \Delta t_{j}} \phi_{j}\right\rangle \\
& =\int \prod_{k}\left(\prod_{j=1}^{n-1} d \bar{\phi}_{j} d \phi_{j}\right) \prod_{j=0}^{n-1} e^{\left.-\left[\frac{1}{2}\left(\bar{\phi}_{j+1} \frac{\phi_{j+1}-\phi_{j}}{\Delta t_{j}}-\frac{\bar{\phi}_{j+1}-\bar{\phi}_{j}}{\Delta t_{j}} \phi_{j}\right)+i \omega_{k} \bar{\phi}_{j+1} \phi_{j}\right)\right] \Delta t_{j}}
\end{aligned}
$$

In this manipulation, we handled the time ordering by inserting a sequence of complete sets and used the fact that coherent states remain coherent under time evolution. To avoid proliferation of indices, we suppressed indices $k$ on $\phi$ and $\bar{\phi}$; the remaining indices label the time slices, with the understanding that times 0 and $n$ replaces $i$ and $f$. Taking $\Delta t_{j}=t_{j+1}-t_{j} \rightarrow 0$ and using the notation $\dot{\phi}=\frac{\phi_{j+1}-\phi_{j}}{\Delta t_{j}}$ we find

$$
U\left(\bar{\phi}_{f} \mid \phi_{i}\right)=\int \mathcal{D} \phi\left[e^{\frac{1}{2}\left(\bar{\phi}_{f} \phi_{f}+\bar{\phi}_{i} \phi_{i}\right)} e^{-\int d t\left[\frac{1}{2}(\bar{\phi} \dot{\phi}-\dot{\bar{\phi}} \phi)+i \omega \bar{\phi} \phi\right]}\right] e^{-\frac{1}{2}\left(\bar{\phi}_{f} \phi_{f}+\bar{\phi}_{i} \phi_{i}\right)}
$$

in which, of course, we have introduced a new notation for the measure. The purpose of the additional, vacuous exponentials will be explained shortly.

A Gaussian integral worthy of the name should be calculated exactly using the stationary phase method. Here a first cut at such an evaluation would involve imposing the equations of motion found by varying $\bar{\phi}$ and $\phi$ separately ${ }^{\star}$. However to obtain a precise prescription the correct measure must be kept in mind. That measure included only variables with indices 1 through $n-1$, so in applying the stationary phase method we must not allow variations at the end points. In the given variables this is an awkward constraint, because as we vary $\bar{\phi}$ with

^ We ignore the determinant, which does not depend on the boundary conditions 
$\bar{\phi}_{f}$ fixed we inevitably vary $\bar{\phi}_{i}$, and similarly for the holomorphic variables. By adjusting the boundary term appropriately, one is able to organize the expression as in (2.10), where the expression in the square brackets allows the full variation. Indeed, upon translating back to the discrete notation, one easily checks that the bracketed expression contains neither $\phi_{f}$ nor $\bar{\phi}_{i}$. Evidently the symbols with those names should be considered functions of the other boundary conditions, not as independent parts of the path integral. By way of contrast, in the factor outside the brackets, the bar really does denote complex conjugation.

The expression in the square brackets is the standard expression for the evolution operator in the holomorphic representation. Finding and integrating the equations of motion, one readily calculates

$$
U\left(\bar{\phi}_{f} \mid \phi_{i}\right)=\prod_{k}\left[e^{\bar{\phi}_{f} e^{-i \omega_{k}\left(t_{f}-t_{i}\right)} \phi_{i}}\right] e^{-\frac{1}{2}\left(\bar{\phi}_{f} \phi_{f}+\bar{\phi}_{i} \phi_{i}\right)}
$$

This expression could also be derived directly, using simple properties of coherent states.

Taking the initial state to be vacuum, i.e. $\phi_{i}=0$, we find the vacuum wave functional to be a simple normalized Gaussian. This final result should come as no surprise, because the Hamiltonian is zero acting on the vacuum, so $U\left(\bar{\phi}_{f} \mid 0\right)=\left\langle\bar{\phi}_{f} \mid 0\right\rangle$, leaving only the normalization of the coherent state. This satisfying result exhibits also, unfortunately, the essential triviality of the procedure: the insertion of intermediate states, and subsequent integration, is vacuous. The entire calculation effectively occurs at one time slice, and one does not find the Lorentz or conformal symmetries exhibited explicitly. 


\subsection{Feynman's Path Integral}

To exhibit these symmetries, we would like to find a form of the path integral that, written in real space, resembles the one derived from geometrical considerations. For this purpose, we return to the original Feynman construction of the path integral. The Hamiltonian is

$$
\hat{H}=\int \frac{d k}{2 \pi} \omega_{k} \hat{\phi}_{k}^{\dagger} \hat{\phi}_{k}=\int \frac{d k}{2 \pi} \frac{1}{2 \omega_{k}}\left(\hat{p}_{k}^{2}+\omega_{k}^{2} \hat{q}_{k}^{2}\right)
$$

where $\hat{\phi}_{k}=\frac{\omega_{k} \hat{q}_{k}+i \hat{p}_{k}}{\sqrt{2 \omega_{k}}}$ and $\hat{\phi}_{k}^{\dagger}=\frac{\omega_{k} \hat{q}_{k}-i \hat{p}_{k}}{\sqrt{2 \omega_{k}}}$, so that $\left[\hat{\phi}_{k}, \hat{\phi}_{k}^{\dagger}\right]=2 \pi \delta\left(k-k^{\prime}\right)$. As a basis in the Hilbert space we introduce the overcomplete set $|q\rangle$ with the property $\hat{q}_{k}|q\rangle=q_{k}|q\rangle$. From the commutation relations $\left[\hat{p}_{k}, \hat{q}_{k}\right]=-2 \pi i \delta\left(k-k^{\prime}\right)$ we derive that $\hat{p}_{k}$ act on such states as $\hat{p}_{k}|q\rangle=i \frac{d}{d q_{k}}|q\rangle$. Introducing also eigenstates of the momentum operator we find $\langle p \mid q\rangle=e^{-i p_{k} q_{k}}$. The operators $\hat{q}_{k}$ and $\hat{p}_{k}$ are Hermitean so their eigenvalues are real. From the Hamiltonian we see that vacuum is defined by $\hat{\phi}_{k}|0\rangle=0$, and integrate to find

$$
\Psi[q]=\langle q \mid 0\rangle \propto \prod_{k} e^{-\frac{\omega_{k}}{2} q_{k}^{2}}
$$

Normalizing the measure so $\int d q_{k} e^{-\omega q_{k}^{2}} \equiv 1$,

$$
\int d q|q\rangle\left\langle q\left|=1=\int d p\right| p\right\rangle\langle p|
$$

(we omit the indices and denote the numbers $q_{k}$ collectively as $q$, and similarly for 
$\left.p_{k}\right)$, we are ready to derive the path integral:

$$
\begin{aligned}
U\left(q_{f} \mid q_{i}\right) & \equiv\left\langle q_{f}\left|T e^{-i \hat{H} t}\right| q_{i}\right\rangle \\
& =\int \prod_{j=1}^{n-1} d q_{j} \prod_{j=0}^{n-1} d p_{j}\left\langle q_{j+1} \mid p_{j}\right\rangle\left\langle p_{j}\left|e^{-i \hat{H} \Delta t_{j}}\right| q_{j}\right\rangle \\
& =\int \prod_{j=1}^{n-1} d q_{j} \prod_{j=0}^{n-1} d p_{j} e^{i p_{j}\left(q_{j+1}-q_{j}\right)} e^{-\frac{i}{2}\left(p_{j}^{2}+\omega_{k}^{2} q_{j}^{2}\right) \Delta t_{j}} \\
& \equiv \int \mathcal{D} q \prod_{k} e^{i \int d t \frac{1}{2}\left(\dot{q}_{k}^{2}-\omega_{k}^{2} q_{k}^{2}\right)} \\
& =\int \mathcal{D} \phi e^{i \int d t \frac{1}{2}\left(\dot{\phi}^{2}-(\nabla \phi)^{2}\right)}
\end{aligned}
$$

In this expression, we have defined $t_{n}=t_{f}, t_{0}=t_{i}$, and $\Delta t_{j}=t_{j+1}-t_{j}$, and used a continuum notation.

We have gone into some detail here so that the main point comes out clearly and unambiguously, as follows. By construction, the variables $q_{k}$ are real. This property must hold even it the field is chiral, that is if the $k$ are restricted to be positive (or negative). The classical field includes both components nevertheless, because that is the only way it can be real. All this conforms with our earlier remarks on the geometric derivation of the path integral. We realised in that context that one must allow both left and right movers in the intermediate configurations, so as to work in a set that is closed under dilation of time.

To evaluate the integral, we write $\phi=\phi_{\mathrm{cl}}+\delta \phi$ where $\phi_{c l}$ is a solution of the equation of motion with the specified boundary conditions, while $\delta \phi$ vanishes on the boundary but is otherwise arbitrary. Inserting this decomposition in the path integral, one easily sees that the cross terms disappear. Hence the path integral factorizes, with only one factor depending on the boundary conditions. And that factor is simply the integrand in the path integral, i.e. exponential of the classical action, evaluated for the field $\phi_{c l}$.

It is easy to calculate the classical field even for general boundary conditions, i.e. $\phi$ fixed to be $\phi_{f}$ at $t_{f}$ and $\phi_{i}$ at $t_{i}$. We are especially interested in the special 
case where the initial state is the vacuum. This case is most easily handled by noticing that taking $t_{f}-t_{i} \rightarrow-i \infty$ the Hamiltonian projects on to the ground state. Indeed, finding the classical field and taking this limit, with $\phi_{i}$ fixed at some finite value, has the same effect as removing the components that approach $\infty$ in the limit, or alternatively requiring that the classical field satisfies $\phi \rightarrow 0$ in this limit. Hence the wave functional for the vacuum state is calculated simply by imposing $\phi \rightarrow 0$ as $t \rightarrow-i \infty$ on the classical field.

To tie up this discussion let us relate this careful explicit evaluation, with an identified measure $\prod_{k} d q_{k}$ and a definite prescription for calculation of the path integral, to the heuristic (2.3). We shall illustrate this by reference to the wave functional for a chiral field in $1+1$ dimensional Euclidean space. We find the classical field that satisfy the equations of motion and the appropriate boundary conditions, and calculate the path integral for that one configuration. The vacuum state is identified by the boundary condition $\phi \rightarrow 0$ as $t \rightarrow-i \infty$ or, in Euclidean space, $\phi \rightarrow 0$ as $\tau \rightarrow-\infty$. Imposing the reality condition, we write

$$
\phi_{\mathrm{cl}}=\int_{-\infty}^{\infty} \frac{d k}{\sqrt{4 \pi|k|}}\left[\phi_{k} e^{k z}+\bar{\phi}_{k} e^{k \bar{z}}\right]
$$

Now we select the vacuum by restricting the integral to positive $\mathrm{k}$. Calculating the action for this field configuration, we find

$$
A_{\mathrm{cl}}\left(\phi_{\mathrm{cl}}\right)=\frac{1}{2 \pi} \int \partial \phi_{\mathrm{cl}} \bar{\partial} \phi_{\mathrm{cl}}=\frac{1}{2} \int_{0}^{\infty} \frac{d k}{2 \pi} \bar{\phi}_{k} \phi_{k}
$$

The action integral was over the $\tau<0$ half plane. Finally we write the wave functional

$$
\Psi[\phi]=\prod_{k} e^{-\frac{1}{2} \bar{\phi}_{k} \phi_{k}}
$$

a result that coincides with the one found above in other ways. 


\subsection{The Path Integral for Fermions}

With these experiences in mind, let us turn to the question of finding a useful prescription for the fermion path integral. For free fermions the Hamiltonian is again of the form $\hat{H}=\omega a^{\dagger} a$, but the creation and annihilation operators satisfy anticommutation relations $\left\{a, a^{\dagger}\right\}=1$. Introducing hermitean variables $\hat{q}=\frac{a+a^{\dagger}}{\sqrt{2}}$ and $\hat{p}=\frac{i\left(a^{\dagger}-a\right)}{\sqrt{2}}$, we find $\{\hat{p}, \hat{q}\}=0$. This relation does not allow for a realization wherein $\hat{q}$ is diagonal and $\hat{p}$ is expressed as a derivative operator. That would require a nontrivial (anti)commutation relation. Thus the road leading to the Feynman path integral appears to be closed, and we must fall back on a version of the holomorphic integral. Indeed, one can introduce a basis that diagonalize $a$ and represent $a^{\dagger}$ as an anticommuting derivative. This leads us to the holomorphic representation of the path integral, with the additional subtlety that the holomorphic variables are anticommuting, so that in manipulating the expressions we must take care of their order. In the bosonic (second-order) case this form of the path integral was rather trivial and unsatisfying geometrically, but as we shall now demonstrate the situation is quite different in the fermionic (first-order) case.

As usual, we consider a massless field in two dimensions. Introducing coherent states

$$
|\psi\rangle=\prod_{k} e^{-\frac{1}{2} \bar{\psi}_{k} \psi_{k}} e^{\hat{\psi}_{k}^{\dagger} \psi_{k}}|0\rangle
$$

we preserve the normalization of the state $\langle\psi \mid \psi\rangle$ and the resolution of the identity

$$
\int \prod_{k} d \bar{\psi}_{k} d \psi_{k}|\psi\rangle\langle\psi|=1
$$


Since the Hamiltonian remains $\hat{H}=\int \frac{d k}{2 \pi}|k| \hat{\psi}_{k}^{\dagger} \hat{\psi}_{k}$ we find as before

$$
\begin{aligned}
U\left(\bar{\psi}_{f} \mid \psi_{i}\right) & =\int \prod_{k}\left(\prod_{j=1}^{n-1} d \bar{\psi}_{j} d \psi_{j}\right) e^{\left.-\left[\frac{1}{2}\left(\bar{\psi}_{j+1} \frac{\psi_{j+1}-\psi_{j}}{\Delta t_{j}}-\frac{\bar{\psi}_{j+1}-\bar{\psi}_{j}}{\Delta t_{j}} \psi_{j}\right)+i|k| \bar{\psi}_{j+1} \psi_{j}\right)\right] \Delta t_{j}} \\
& \equiv \int \mathcal{D} \psi \prod_{k} e^{\int d t \frac{1}{2}[\dot{\bar{\psi}} \psi-\bar{\psi} \dot{\psi}]+i|k| \bar{\psi} \psi} \\
& \equiv \int \mathcal{D} \psi e^{-\frac{1}{4 \pi} \int d t d \sigma \psi\left(\partial_{t}-\partial_{\sigma}\right) \psi}
\end{aligned}
$$

In transforming back to real space we have introduced

$$
\psi=\int \frac{d k}{2 \pi}\left[\psi_{k} e^{-i k \sigma}+\bar{\psi}_{k} e^{i k \sigma}\right]
$$

which by construction is real. We see that for fermions, unlike for bosons, the holomorphic path integral leads to the invariant form that we know from the geometrical derivation. However here as before these expressions are a little subtle. In particular, in deriving and interpreting them one may be tempted to give up the reality condition on the field, in which case one must take boundary terms carefully into account in evaluating them, and a trivial integral is found.

To evaluate the path integral without giving up the reality condition we proceed as for bosons and write the most general field $\psi=\psi_{\mathrm{cl}}+\delta \psi$, where $\psi_{\mathrm{cl}}$ satisfies the boundary conditions on $\psi$ while $\delta \psi$ is 0 on the boundaries, that is the initial and final time slices. Finally, choose $\psi_{\mathrm{cl}}$ to be the unique real field that satisfies the boundary conditions, as well as the Klein-Gordon (not the Weyl-Dirac) equation. (Any field with the proper boundary conditions might have been used; the KleinGordon equation is imposed only to insure uniqueness!). Now note that in

$$
\frac{1}{4 \pi} \int \psi\left(\partial_{t}-\partial_{\sigma}\right) \psi=\frac{1}{4 \pi} \int \psi_{\mathrm{cl}}\left(\partial_{t}-\partial_{\sigma}\right) \psi_{\mathrm{cl}}+\frac{1}{4 \pi} \int \delta \psi\left(\partial_{t}-\partial_{\sigma}\right) \delta \psi
$$

mixed terms do not enter, due to the reality condition on the field and the boundary conditions on $\delta \psi$. The path integral measure $\mathcal{D} \psi$ is invariant under translations 
with a fixed function $\psi_{\mathrm{cl}}$, so we might as well take it to be $\mathcal{D} \delta \psi$. Then the path integral factorizes. The fluctuation part is independent of the boundary conditions and can be omitted. We find that the path integral can be calculated by taking the action of the field configuration $\psi_{\mathrm{cl}}$, as advertized. At no point in the present derivation did we use a variational principle - specifically, we never found that we should impose the equation of motion on the classical field.

We have found that the fermionic path integral is naturally expressed using real classical fermion fields to parametrize the initial and final states, thus putting them on an equal footing. The interpretation of such an amplitude between real states is not a priori clear, because the original problem did not have real fields. It is implicit in our derivation that the path integral expresses an amplitude between coherent states. To find the ket-coherent state that corresponds to a given real wave function, take the spatial Fourier transform. The components with positive wave vector provides the eigenvalues of the elementary oscillators of the Weyl field. Conversely, the real classical field corresponding to given eigenvalues of the elementary oscillators is found by using the eigenvalues as Fourier components with positive $\mathrm{k}$-vectors, and their complex conjugates for the negative k-vectors. Thus the amplitudes that are expressed by our unorthodox path integral are exactly the same as those that are expressed by the more conventional holomorphic path integral.

Just as for bosons, on taking $t_{i} \rightarrow-i \infty$ with $\psi_{i}$ fixed we find that $\psi_{i}$ disappears from the problem and is replaced by the requirement that $\psi_{\mathrm{cl}} \rightarrow 0$ as $t \rightarrow-i \infty$. This is a very convenient characterization of vacuum. As a final step in elucidating the fermionic path integral, let us Euclideanize the classical field and the action. To obtain expressions that resemble those found previously for bosons, we require that the Euclidean classical field is real; that is, that it is a sum of a holomorphic function and an antiholomorphic one. We then have, finally,

$$
\Psi[\psi]=e^{-\frac{1}{2 \pi} \int \psi \bar{\partial} \psi} .
$$

It is easy to verify that the exponent is real. In this form, conformal invariance is 
manifest.

To exemplify the use of this machinery, let us calculate the vacuum wave functional. We proceed as for bosons: write the classical field

$$
\psi_{\mathrm{cl}}=\int_{0}^{\infty} \frac{d k}{\sqrt{2 \pi}}\left[\psi_{k} e^{k z}+\bar{\psi}_{k} e^{k \bar{z}}\right]
$$

where we have imposed the reality condition, and the condition that the field vanishes at early times. Inserting this in the action we find

$$
A\left(\psi_{\mathrm{cl}}\right)=\frac{1}{2 \pi} \int \psi_{\mathrm{cl}} \bar{\partial} \psi_{\mathrm{cl}}=\frac{1}{2} \int \frac{d k}{2 \pi} \bar{\psi}_{k} \psi_{k}
$$

and for the wave functional

$$
\Psi[\psi]=\prod_{k>0} e^{-\frac{1}{2} \bar{\psi}_{k} \psi_{k}}
$$

Thus this formalism indeed yields the expected $\langle 0 \mid \psi\rangle$.

\section{Geometric Entropy of Free Bosons and Fermions in 2 Dimensions}

\subsection{Strategy}

We now return to the problem that motivated the preceding ordeal. We will use the formalism developed to carry out the calculation of the geometric entropy for massless scalar bosons and spin $\frac{1}{2}$ fermions in $1+1$ dimensions. In this context, the essential problem is the entropy associated with a half-line. We will demonstrate that each step in the calculation of the entropy can be carried out very explicitly, using the flexibility of the path integral expressions just developed. This may not be the most efficient way to reach that specific goal, but it provides an explicit, and hopefully transparent, derivation of the entropy in a manner that is parallel for fermions and bosons, and capable of generalization. 
The first step is to calculate the vacuum wave functional

$$
\Psi[R, L] \propto \int \mathcal{D} \phi e^{-A(\phi)} .
$$

Here the path integral is over all fields that satisfy

$$
\phi(\sigma)=\theta(\sigma) R(\sigma)+\theta(-\sigma) L(\sigma)
$$

at a time slice taken to be $\tau=0$, and we also impose

$$
\phi(x) \rightarrow 0 \quad \tau \rightarrow-\infty
$$

to project onto the vacuum. In earlier sections we worked with the Fourier components of the field but these are not localized to be either on the left hand side of the axis, or on the right hand side. Hence we need to choose as a basis instead functions that are partly localized, but still resemble Fourier modes sufficiently to approximately diagonalize the action. Wavelets [14] are designed for exactly this purpose, that is to provide wave-like functions with compact support. They diagonalize the action approximately, and are likely to be of considerable use in problems with more complicated structure ${ }^{\star}$. For the present however, we will stick to Fourier modes, and simply transform the argument of the fields $R$ and $L$ instead. More precisely, we will introduce a convenient coordinate system that maps both half lines to full lines, for which we can use the standard Fourier transform. The density matrix will not quite diagonalize, but will break up into $2 \times 2$ blocks. This trick is in essence due to Unruh [10]. The boundary conditions have a unique

$\star$ There is also a more fundamental point that ought to be mentioned in this context. The geometric entropy as defined corresponds to the density matrix for a hypothetical experimenter who has complete access to arbitrarily high frequency modes on the outside, but no access to the inside. A more realistic idealization would be to allow access to low-frequency modes on the outside only, tracing over both very high frequencies in general and also low frequencies on the inside. These notions could be formalized using wavelets. The entropy thus defined would be finite, diverging only as the limiting frequency is taken to infinity. 
solution among fields that are the sum of a holomorphic and an anti-holomorphic piece. That field is the classical field and the path integral is calculated by finding the action of the classical field. This is true, as we have seen, for bosons and fermions alike.

Having obtained the wave functional in a convenient basis, the next step is to sum over the left variables, and then to find the entropy corresponding to the resulting density matrix. This will be done using a replica trick, as in [4].

We will use the complexification $z=\sigma+i \tau$ and $\bar{z}=\sigma-i \tau$. Since $\tau$ is the Euclidean time this amounts simply to the light cone coordinates. This convention interchanges $\tau$ and $\sigma$ compared to the one conventional in the string theory literature.

\subsection{Classical Fields}

First we calculate the classical field for bosons. $\phi(x)$ is specified at $\tau=0$; our task is to determine $\phi(x)$ in the entire lower half plane. We write

$$
\phi(z, \bar{z})=\frac{i}{2 \pi} \int_{-\infty}^{\infty} d w\left(\frac{1}{w-z}-\frac{1}{w-\bar{z}}\right) \phi(w)
$$

In the integral $w$ is a real variable. This integral equation is clearly valid on the real line, and extends by regularity to the entire negative half-plane (it is just the usual Poisson integral for this problem). We change of variables according to

$$
w=\operatorname{sign}(w) e^{x}, \quad z=e^{\eta}, \quad \bar{z}=e^{\bar{\eta}},
$$

leaving the field untouched. The field is defined in the lower half plane, so in inverting $z=e^{\eta}$ we must choose $\operatorname{Im} \eta \leq 0$. Thus the positive half-axis is mapped to the entire real axis, and the negative half-axis is mapped to the line with imaginary 
part $-i \pi$. We write

$$
R(x)=\int \frac{d \omega}{\sqrt{4 \pi|\omega|}} e^{-i \omega x} r_{\omega} \quad L(x)=\int \frac{d \omega}{\sqrt{4 \pi|\omega|}} e^{-i \omega x} l_{\omega},
$$

thus parametrizing the functions $R$ and $L$ by their Fourier components in the transformed variable. The reality condition on the field $\phi$ is expressed as $r_{\omega}=\bar{r}_{-\omega}$ and $l_{\omega}=\bar{l}_{-\omega}$. Now we have

$$
\phi(\eta)=\frac{i}{2 \pi} \int \frac{d \omega}{\sqrt{4 \pi|\omega|}} \int_{-\infty}^{\infty} d x\left[\left(\frac{e^{-i \omega x}}{1-e^{\eta-x}} r_{\omega}-\frac{e^{-i \omega x}}{1+e^{\eta-x}} l_{\omega}\right)-\text { h.c. }\right]
$$

and calculating the integrals over $x$ by contour integration and recalling $\operatorname{im} \eta \leq 0$ we find

$$
\phi(\eta)=\int \frac{d \omega}{\sqrt{4 \pi|\omega|}}\left[e^{-i \omega \eta} \frac{1}{2 \operatorname{sh} \pi \omega}\left(e^{\pi \omega} r_{\omega}-l_{\omega}\right)-e^{-i \omega \bar{\eta}} \frac{1}{2 \operatorname{sh} \pi \omega}\left(e^{-\pi \omega} r_{\omega}-l_{\omega}\right)\right] .
$$

It is easy check that indeed (3.2) is satisfied, i.e. that $\phi=R$ for $\eta \in \mathcal{R}$ and $\phi=L$ for $\eta \in \mathcal{R}-i \pi$. Indeed, this expression could easily have been found by writing the general form of the wave function and determining the coefficients from the boundary conditions. In this reasoning, the boundary condition that $\phi \rightarrow 0$ at early times, that is the choice of vacuum, is expressed by imposing regularity throughout the strip. The present, constructive approach has the advantage that it is easily generalized to the case of fermions.

Indeed, let us write

$$
\psi(z, \bar{z})=\frac{i}{2 \pi} \int_{-\infty}^{\infty} d w\left(\frac{1}{w-z}-\frac{1}{w-\bar{z}}\right) \psi(w) .
$$

Let us again introduce the left/right split (3.2), and the change of variables (3.4). Now, however, we transform the fermion field according to

$$
\psi(z, \bar{z})=e^{-\frac{1}{2} \eta} \psi(\eta, \bar{\eta}), \quad z=e^{\eta}, \bar{z}=e^{\bar{\eta}}
$$

The necessity to transform $\psi$, in contrast to $\phi$, ultimately reflects the non-trivial conformal weight of $\psi$. The original $\psi$ was real and that transforms to a real $\psi$ on 
the real axis but to a purely imaginary $\psi$ on $\mathcal{R}-i \pi$. Introducing Fourier transforms

$$
R(x)=\int \frac{d \omega}{\sqrt{2 \pi}} e^{-i \omega x} r_{\omega}, \quad L(x)=\int \frac{d \omega}{\sqrt{2 \pi}} e^{-i \omega x} l_{\omega}
$$

this is expressed by $\bar{r}_{\omega}=r_{-\omega}$ and $\bar{l}_{\omega}=-l_{-\omega}$. We have chosen a different normalization here than in (3.5) for bosons, in order that the Fourier components $r_{\omega}$ and $l_{\omega}$ have mass dimension $-\frac{1}{2}$ for fermions, as it did bosons. Collecting formulae, we have

$$
\psi(\eta, \bar{\eta}) e^{-\frac{1}{2} \eta}=i \int \frac{d \omega}{\sqrt{2 \pi}} \int_{-\infty}^{\infty} \frac{d x}{2 \pi} e^{-i \omega x} e^{\frac{1}{2} x}\left[\left(\frac{r_{\omega}}{e^{x}-e^{\eta}}-\frac{i l_{\omega}}{e^{x}+e^{\eta}}\right)-(\eta \rightarrow \bar{\eta})\right] .
$$

The extra factors of $e^{\frac{1}{2} x}$ and $e^{\frac{1}{2} \eta}$ compared to the boson case come from the transformation of the fermion fields as a $\left(\frac{1}{2}, 0\right)$ field. This is also the origin of the extra $i$ in the second term. Upon performing the integrals we find

$$
\psi(\eta, \bar{\eta})=\int \frac{d \omega}{\sqrt{2 \pi}}\left[e^{-i \omega \eta} \frac{1}{2 \operatorname{ch} \pi \omega}\left(e^{\pi \omega} r_{\omega}+l_{\omega}\right)+e^{\frac{\eta-\bar{\eta}}{2}} e^{-i \omega \bar{\eta}} \frac{1}{2 \operatorname{ch} \pi \omega}\left(e^{-\pi \omega} r_{\omega}-l_{\omega}\right)\right]
$$

In principle this decomposition could have been found by writing the ansatz,

$$
\psi(\eta, \bar{\eta})=\int \frac{d \omega}{\sqrt{2 \pi}}\left[e^{-i \omega \eta} \psi_{\omega}+e^{\frac{\eta-\bar{\eta}}{2}} e^{-i \omega \bar{\eta}} \bar{\psi}_{\omega}\right]
$$

and determining the coefficients from the boundary conditions. However this ansatz is non-trivial, the $e^{\frac{\eta-\bar{\eta}}{2}}$ being due to the conformal dimensions of the fields. In the present, constructive approach it is well motivated from the transformation properties of the fermion field. 


\subsection{Wave Functionals}

Having found the classical field for both bosons and fermions we proceed to find the wave function. The classical actions are

$$
A_{\mathrm{cl}}^{\mathrm{boson}}=\frac{1}{2 \pi} \int \partial \phi_{\mathrm{cl}} \bar{\partial} \phi_{\mathrm{cl}}=\frac{1}{2} \int_{0}^{\infty} \frac{d \omega}{2 \pi}\left[\frac{\operatorname{ch} \pi \omega}{\operatorname{sh} \pi \omega}\left(\bar{r}_{\omega} r_{\omega}+\bar{l}_{\omega} l_{\omega}\right)-\frac{1}{\operatorname{sh} \pi \omega}\left(\bar{r}_{\omega} l_{\omega}+\bar{l}_{\omega} r_{\omega}\right)\right]
$$

and

$$
A_{\mathrm{cl}}^{\text {fermion }}=\frac{i}{2 \pi} \int \psi_{\mathrm{cl}} \bar{\partial} \psi_{\mathrm{cl}}=\frac{1}{2} \int_{0}^{\infty} \frac{d \omega}{2 \pi}\left[\frac{\operatorname{sh} \pi \omega}{\operatorname{ch} \pi \omega}\left(r_{-\omega} r_{\omega}+l_{-\omega} l_{\omega}\right)+\frac{1}{\operatorname{ch} \pi \omega}\left(r_{-\omega} l_{\omega}-l_{-\omega} r_{\omega}\right)\right]
$$

In the fermion case we avoid the $\bar{l}_{\omega}$ notation to prevent confusion due to the relation $\bar{l}_{\omega}=-l_{-\omega}$. In evaluating these expressions we have used the integrals

$$
\begin{gathered}
\int \partial e^{-i \omega^{\prime} \eta} \bar{\partial} e^{-i \omega \bar{\eta}}=2 \pi \delta\left(\omega+\omega^{\prime}\right) \omega e^{\pi \omega} \operatorname{sh} \pi \omega \\
\int e^{-i \omega^{\prime} \eta} \bar{\partial} e^{\frac{\eta-\bar{\eta}}{2}} e^{-i \omega \bar{\eta}}=2 \pi \delta\left(\omega+\omega^{\prime}\right) e^{\pi \omega} i \operatorname{ch} \pi \omega \\
\int e^{\frac{\eta-\bar{\eta}}{2}} e^{-i \omega^{\prime} \bar{\eta}} \bar{\partial} e^{\frac{\eta-\bar{\eta}}{2}} e^{-i \omega \bar{\eta}}=0 .
\end{gathered}
$$

The wave functionals are simply

$$
\Psi[R, L]=e^{-A_{\mathrm{cl}}}
$$

It is a good check, to verify some of their necessary qualitative properties. In the limit $\omega \rightarrow \infty$ the wave functionals reduce to the results found with no left/right split, that is, Gaussians with the same normalization. This occurs because excitations that are almost localized do not mix with excitations on the other half line. At finite frequencies, there is overlap. We note however that the operator in the 
exponent has the same determinant as previously, as indeed it must for a unitary change of basis. This feature is easily checked using the path integral measure $\prod_{\omega} d l_{-\omega} d l_{\omega} d r_{-\omega} d r_{\omega}$, and the useful integral formula

$$
\int d \bar{z} d z e^{-\bar{z} M z+\bar{z} j+\bar{j} z}=(\operatorname{det} M)^{\mp \frac{1}{2}} e^{\bar{j} M^{-1} j}
$$

where the upper and lower signs refer to bosons and fermions respectively.

\subsection{Geometric Entropy}

To calculate the entropy from the wave functional we must first calculate the density matrix

$$
\rho\left[R, R^{\prime}\right]=\int \mathcal{D} L \bar{\Psi}[R, L] \Psi\left[R^{\prime}, L\right] .
$$

The measure in the integral is simply $\prod_{\omega} d l_{-\omega} d l_{\omega}$, which we have normalized so that a Gaussian gives unity. The wave functional $\Psi$ is normalized by requiring $\operatorname{Tr} \rho=1$ with respect to the same measure. We find

$$
\rho\left[R, R^{\prime}\right]=\prod_{\omega>0} \frac{\operatorname{sh} \pi \omega}{\operatorname{ch} \pi \omega} \exp \left\{-\frac{1}{2 \operatorname{sh} 2 \pi \omega}\left[\operatorname{ch} 2 \pi \omega\left(\left|r_{\omega}\right|^{2}+\left|r_{\omega}^{\prime}\right|^{2}\right)-\left(\bar{r}_{\omega} r_{\omega}^{\prime}+\bar{r}_{\omega}^{\prime} r_{\omega}\right)\right]\right\}
$$

for fermions as well as for bosons. We have defined $\left|r_{\omega}\right|^{2}=r_{-\omega} r_{\omega}$, and use again $r_{-\omega} \equiv \bar{r}_{\omega}$. In verifying this expression it is important to recall that integrals over Grassmann variables gives a determinant in the numerator rather than in the denominator.

Next we want to use the replica trick

$$
S_{\text {geom }}=-\left(1-\frac{d}{d n}\right) \ln \operatorname{Tr} \rho^{n},
$$

so we need to calculate $\rho^{n}$. The result is

$$
\frac{\rho^{n}\left[R, R^{\prime}\right]}{\operatorname{Tr} \rho^{n}}=\prod_{\omega>0} \frac{\operatorname{sh} n \pi \omega}{\operatorname{ch} n \pi \omega} \exp \left\{-\frac{1}{2 \operatorname{sh} 2 \pi n \omega}\left[\operatorname{ch} 2 \pi n \omega\left(\left|r_{\omega}\right|^{2}+\left|r_{\omega}^{\prime}\right|^{2}\right)-\left(\bar{r}_{\omega} r_{\omega}^{\prime}+\bar{r}_{\omega}^{\prime} r_{\omega}\right)\right]\right\}
$$


where

$$
\begin{gathered}
\operatorname{Tr} \rho^{n}=\frac{(2 \operatorname{sh} \pi \omega)^{2 n}}{(2 \operatorname{sh} n \pi \omega)^{2}} \quad \text { (bosons) } \\
\operatorname{Tr} \rho^{n}=\frac{(2 \operatorname{ch} n \pi \omega)^{2}}{(2 \operatorname{ch} \pi \omega)^{2 n}} \quad \text { (fermions) }
\end{gathered}
$$

These formulae can be verified inductively. The difference between bosons and fermions is two-fold. First: Grassmann integrals, as mentioned above, give determinants in the numerator rather than in the denominator. Second: in taking the trace for bosons we simply identify $r_{\omega}=r_{\omega}^{\prime}$ and do the $d r_{-\omega} d r_{\omega}$ integral, but for fermions we must take $r_{\omega}=-r_{\omega}^{\prime}$ instead. This difference has been explained in an elementary way by Soper [15]. We can also understand it simply in our framework, as follows. In the holomorphic formalism the typical bilinear operator can be expanded as a string of variables in the form anti-holomorphic, holomorphic, anti-holomorphic, etc. In taking the trace, however, we pair the last holomorphic variable in a string like this with the first anti-holomorphic variable. For Grassmann variables this operation must be accompanied by a change of sign, which is most easily handled by changing the sign on one of the variables. This accounts for the antisymmetric boundary conditions.

With these expressions the replica trick (3.11) can be carried through to yield

$$
S_{\text {geom }}= \pm 2 \int \frac{d \omega}{2 \pi}\left(1-\omega \frac{d \omega}{d \omega}\right) \ln \left(1 \mp e^{-2 \pi \omega}\right)=4 \int d \omega \frac{\omega}{e^{2 \pi \omega} \mp 1}
$$

for the entropy. The upper sign refers to boson and the lower to fermions. These are simply the thermodynamic expressions for the entropy of a 1-dimensional gas of (spinless) bosons or fermions respectively. So far, the possible values of $\omega$ have been specified informally as $\int \frac{d \omega}{2 \pi}$, and strictly speaking expressions such as $\prod_{\omega}$ did not make sense without regularization. At this point we impose periodic (or anti-periodic) boundary conditions in a box of length $L$, thus arriving at

$$
S_{\text {geom }}=\frac{c}{6} L
$$

with $c=\frac{1}{2}, 1$ for fermions and bosons, respectively. In this formula the length $L$ is 
the length as measured in the transformed coordinate system. Transforming back to the original coordinates and introducing an ultraviolet cutoff $\epsilon$ and an infrared cutoff $\Sigma$ we can write $L=\ln \frac{\Sigma}{\epsilon}$. This result and the interpretation of the ensuing divergence have been discussed extensively from another point of view in [8].

We have now accomplished the technical task we set ourselves, to obtain a path integral sufficiently flexible to allow us to calculate wave functionals and geometric entropy in a straightforward manner, applicable both to bosons and to fermions. We were surprised, that to do so we had to forge some new tools.

Acknowledgements: We wish to thank Curt Callan, who was involved in the genesis of this work, for many helpful discussions. 


\section{REFERENCES}

1. L. Bombelli, R. Koul, J. Lee, and R. Sorkin, Phys. Rev. D34 (1986) 373.

2. C. Holzhey, Princeton University thesis, 1992, (unpublished)

3. M. Srednicki, Phys. Rev. Lett. 71, (1993) 666

4. C.G.Callan and F. Wilczek, On Geometric Entropy, hep-th-9401072

5. J.S.Dowker, Remarks on Geometric Entropy, MUTP-94-2,hep-th-9401159

6. D. Kabat and M.J.Strassler, A Comment on Entropy and Area, RU-9410,hep-th/9401125

7. L. Susskind, Some Speculations About Black Hole Entropy in String Theory, RU-93-44,hep-th/9309145

8. C. Holzhey, F. Larsen and F. Wilczek, Geometric and Renormalized Entropy, PUPT-1454, IASSNS-93/88, hep-th/9403108

9. L. Susskind and J. Uglum, Black Hole Entropy in Canonical Quantum Gravity and Superstring Theory, SU-ITP-94-1, hep-th/9401070

10. W. G. Unruh, Phys. Rev. D14 (1977) 870

11. J. L. Cardy, Conformal Invariance and Statistical Mechanics, in Fields, Strings, and Critical Phenomena Les Houches, Session XLIX, 1988, eds. E.Brézin and J. Zinn-Justin.

12. L. Faddeev and Slavnov, Gauge Fields, Introduction to Quantum Theory, Benjamin/Cummings (1980)

13. C. Itzykson and J. B. Zuber, Quantum Field Theory, McGraw Hill (1980)

14. M. B. Ruskai et al., Wavelets and Their Application, Jones and Bartlett (1992)

15. D. E. Soper, Phys. Rev. D18(1978) 4590 